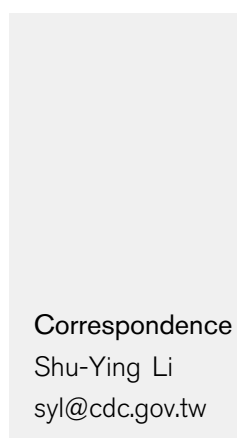

Received 20 January 2006

Accepted 15 March 2006

\section{Surveillance of invasive Streptococcus pneumoniae in Taiwan, 2002-2003}

\author{
Ying-Yan Chen, Shu-Man Yao, Chen-Ying Chou, Yi-Ching Chang, \\ Pei-Wun Shen, Chung-Ter Huang, Hsun-Pi Su and Shu-Ying Li
} Laboratory for Bacteriology and Mycology, Division of Laboratory Research and Development,
Center for Disease Control, No. 161, Kun-Yang Street, Taipei, Taiwan

\begin{abstract}
A total of 522 Streptococcus pneumoniae invasive isolates from diverse sources were collected from January 2002 to December 2003 in Taiwan in order to understand the serotype distribution of invasive isolates in Taiwan. The most frequently isolated serotypes of $S$. pneumoniae were types 14 $(18 \cdot 4 \%), 23 \mathrm{~F}(15 \cdot 1 \%), 3(13 \cdot 8 \%), 19 \mathrm{~F}(13 \cdot 4 \%), 6 \mathrm{~B}(8 \cdot 2 \%), 9 \mathrm{~V}(3 \cdot 6 \%)$ and $4(2 \cdot 5 \%)$. The majority of cases were either under 5 years of age $(24 \cdot 1 \%)$ or older than 65 years $(36 \cdot 6 \%)$. Serotype distribution in adults aged over 14 years and children aged under 2 years was similar, except for that of type 3 , which was more prevalent in adults. Penicillin-non-susceptible strains accounted for $67 \cdot 7 \%$ of all strains and were the predominant strains of serotypes $23 \mathrm{~F}, 19 \mathrm{~F}, 6 \mathrm{~B}$ and 14. Most strains were susceptible to cephem drug, $85 \cdot 7 \%$ of isolates were susceptible to cefotaxime and $92.9 \%$ were susceptible to ceftriaxone. A total of $72.6 \%$ (379/522) of the isolates were resistant to at least two antibiotics. The $23-$ valent vaccine in the current commercial market would cover $87 \cdot 2 \%$ of the serotypes and $100 \%$ of the penicillin-non-susceptible serotypes of $S$. pneumoniae in Taiwan. The coverage of 7- and 11-valent protein conjugate vaccines of the serotypes in children under 2 years of age would be 78.8 and $86.5 \%$, respectively. These results will help to assess the adequacy of the vaccine formulations marketed in Taiwan.
\end{abstract}

\section{INTRODUCTION}

Streptococcus pneumoniae is the most common cause of community-acquired pneumonia. Infection of $S$. pneumoniae can cause invasive diseases such as bacteraemia, sepsis and meningitis and respiratory diseases such as pneumonia, otitis media and sinusitis, especially in children and the elderly. An increasing number of strains resistant to antimicrobial agents and now endemic in many countries reflects an uncontrolled use of antibiotics (Schito \& Felmingham, 2005; Song et al., 2004). The same tendency is paralleled in Taiwan (Chen et al., 2003; Chiou et al., 1998; Hsueh et al., 1996, 2003; Lo et al., 2003; Shi et al., 1998; Siu et al., 2002), where choosing appropriate antibiotics has become a therapeutic challenge. Interest in the prevention of pneumococcal infections by vaccination has been revived (Whitney et al., 2000, 2003). Since 1983, a 23 -valent vaccine covering 23 serotypes of $S$. pneumoniae has been commercially available and is recommended for people over 65 years of age and for immunocompromised individuals. For children under 2 years, a 7 -valent protein conjugate vaccine is on the market (Baltimore, 1992; Robbins \& Schneerson, 1990). The 23-valent and the 7-valent conjugate vaccines against pneumococcal infection were introduced in Taiwan in 1998 and 2005, respectively. As there are discrepancies in the frequency of distribution among different countries and some serotypes may possess distinct antibiotic-resistance or epidemiological properties (Hausdorff et al., 2005), it is of great interest to continue to monitor the distribution of $S$. pneumoniae serotypes and antibiotic resistance in Taiwan. The surveillance of serotype distribution will help to establish local epidemiological profiles and is important for assessment of the potential applicability of vaccine formulations.

\section{METHODS}

Specimens or isolates of $S$. pneumoniae were obtained from normally sterile sites and reported by the hospitals that voluntarily participate in the reporting system. The age, sex and clinical presentation of each patient were reported. The symptoms included pneumonia, meningitis, bacteraemia, sepsis, arthritis, osteomyelitis, endocarditis, pericarditis and peritonitis. The 28 hospitals ranked at the level of medical centre or regional hospitals from different geographic regions of Taiwan that participated in the study were estimated to provide in-patient service to approximately $27 \%$ of Taiwan's population.

A total of 522 bacterial strains collected from cases conforming to the case definition of invasive pneumococcal disease were cultured on blood agar plates. These S. pneumoniae isolates were grown on trypticase soy agar plates (BBL; Becton Dickinson Microbiology Systems) with $3-5 \%$ sheep blood and incubated at $37^{\circ} \mathrm{C}$ in $5 \% \mathrm{CO}_{2}$ overnight. Alpha-haemolytic colonies exhibiting sufficient morphological characteristics of $S$. pneumoniae were isolated. Identification of 
these isolates as S. pneumoniae was confirmed using Gram staining, the optochin growth inhibition test (Difco Laboratories) and bile solubility testing.

The serotype of each pneumococcal isolate was determined by the Quellung reaction with antisera produced by the Statens Serum Institut (Copenhagen, Denmark).

Antibiotic susceptibility tests were performed using VITEK 2 susceptibility tests (bioMérieux) and resistance was defined according to the National Committee for Clinical Laboratory Standards criteria. For these tests, S. pneumoniae was grown for 18-24 h in trypticase soy agar containing $5 \%$ defibrinated sheep blood (BBL) in $5 \% \mathrm{CO}_{2}$. Bacterial cells were suspended in $0 \cdot 45 \%$ saline, adjusted to the turbidity of a $0 \cdot 5$ McFarland standard and loaded in the AST-GP62 test cards for the VITEK 2 test. The test cards were inserted into the prototype VITEK 2 incubator reader, which gave an interpretation of the test results.

To analyse the differences in serotypes between age groups or in antimicrobial susceptibility, a $\chi^{2}$ test with Yate's correction or Fisher's exact test was performed. EPI INFO (version 6.0) was used to calculate the odds ratios (OR) and $95 \%$ confidence limits. Statistical significance was defined as a value of $P<0 \cdot 05$.

\section{RESULTS}

A total of 522 isolates of invasive S. pneumoniae together with the basic information from patients were collected during a 2-year surveillance period from January 2002 to December 2003. Isolates were obtained from 28 hospitals ranked at the level of medical centre or regional hospitals from different geographic regions of Taiwan: north (13), middle (10), south (3) and east (2). The major specimen types collected were blood ( 482 cases, $92 \cdot 3 \%$ ), cerebrospinal fluid ( 16 cases, $3 \cdot 1 \%)$, pleural effusion (15 cases, $2 \cdot 9 \%)$, ascites (four cases, $0 \cdot 8 \%$ ) and sputum (five cases, $1 \cdot 0 \%$ ).

The age distribution of the 522 invasive $S$. pneumoniae cases was as follows: under 2 years, 52 cases; $3-5$ years, 74 cases; 6-14 years, 21 cases; $15-44$ years, 69 cases; $45-64$ years, 96 cases; 65 years and over, 191 cases; and unknown, 19 cases.

The serotype distribution analysis demonstrated that 26 serotypes were identified within the 522 cases of S. pneumoniae, with the most common serotypes in decreasing order of frequency being: 14 ( 96 cases), $23 \mathrm{~F}$ ( 79 cases), 3 ( 72 cases), 19F (70 cases), 6B (43 cases), 9V (19 cases), 4 (13 cases) and 11 (10 cases) (Fig. 1 and Table 1).

For people aged 65 years and over, the most frequent serotypes were: $14(17 \cdot 3 \%), 3(16 \cdot 8 \%), 19 \mathrm{~F}(16 \cdot 2 \%), 23 \mathrm{~F}$ $(13 \cdot 1 \%), 6 \mathrm{~B}(6 \cdot 3 \%)$ and $9 \mathrm{~V}(3 \cdot 1 \%)$, and in this group, a higher crude mortality rate was also observed. For infants under 2 years of age, the most frequent serotypes were 14 $(28 \cdot 8 \%), 23 \mathrm{~F}(17 \cdot 3 \%), 6 \mathrm{~B}(13 \cdot 5 \%), 19 \mathrm{~F}(11 \cdot 5 \%)$ and 3 $(7 \cdot 7 \%)$. Serotype distribution in adults aged over 14 years and in children aged less than 2 years was similar except for that of type 3 , which was $17 \cdot 4$ and $7 \cdot 7 \%$, respectively. Type 3 was significantly more prevalent in adults over 14 years. Type 14 was significantly more prevalent in children under 14 years. The clinical outcomes after infection also varied and were mainly pneumonia $(42 \cdot 3 \%)$, bacteraemia $(21 \cdot 5 \%)$, sepsis $(15 \cdot 7 \%)$ and meningitis $(3 \cdot 5 \%)$. The crude mortality rate was $14 \cdot 4 \%$ from our results, with types $6 \mathrm{~B}$ and 3 being the most frequent serotype $(13.3$ and $20.0 \%$, respectively) in mortality cases.

Among the serotypes of $S$. pneumoniae isolates from the 522 cases, the coverage rate of the 23 -valent polysaccharide vaccine was $87 \cdot 2 \%$ and that of the 7 -valent conjugate vaccine was $63.0 \%$. The coverage rates varied among different age groups. The coverage rate of the 7 -valent vaccine in children under 2 years old was $78 \cdot 8 \%(41 / 52)$ and in people over 3 years old was $61 \cdot 2 \%(276 / 451)$. In children under 14 years, the coverage rate of the 23 -valent vaccine was $87 \cdot 1 \%(128 / 147)$ and of the 7 -valent conjugate vaccine was $76 \cdot 2 \%(112 / 147)$. In adults older than 14 years, the coverage rate of the 23 -valent vaccine was $87 \cdot 6 \%(312 / 356)$ and of the 7 -valent conjugate vaccine was $57 \cdot 6 \%(205 / 356)$. For children under 2 years old, the coverage rate of the 23 -valent vaccine was as high as $88 \cdot 5 \%(46 / 52)$; however, the protection of the 23 -valent polysaccharide vaccine is not sufficient because of its inability to induce a T-celldependent immune response and the 7-valent conjugate vaccine has to be used instead, with a coverage rate of $78 \cdot 8 \%$ as above.

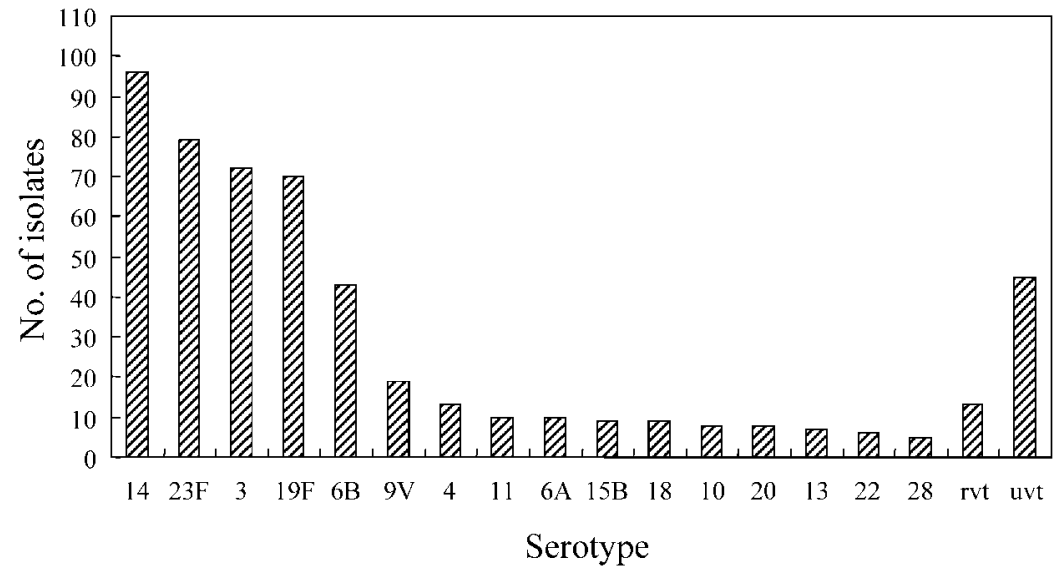

Fig. 1. Serotype distribution of invasive S. pneumoniae disease in Taiwan in samples collected from January 2002 to December 2003. rvt, Other vaccine-related types; uvt, other vaccine-unrelated types. 


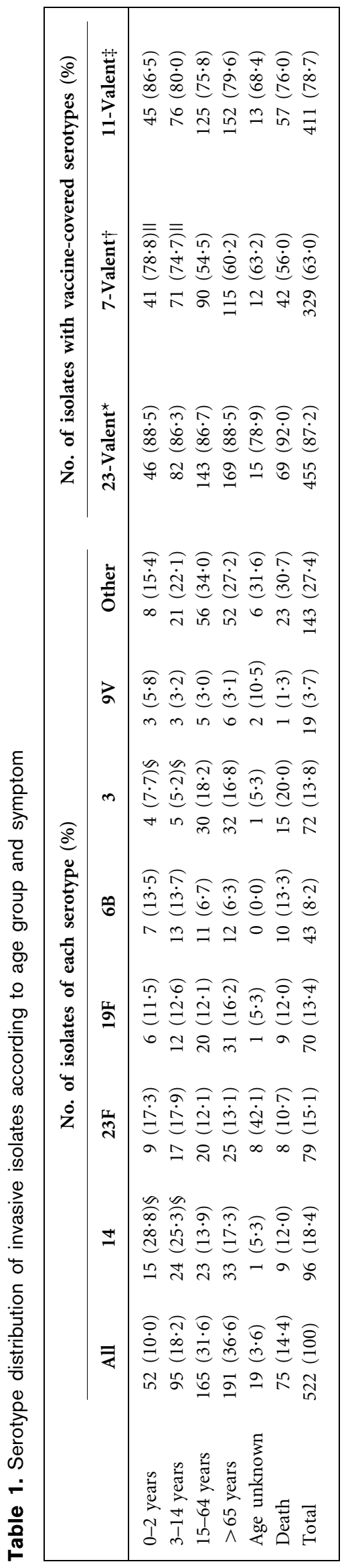

As shown in Table 2, the isolates showed high levels of drug resistance to tetracycline $(84 \cdot 7 \%, 442)$, erythromycin $(78.4 \%, 409)$, trimethoprim/sulfamethoxazole $(52 \cdot 3 \%$, $274)$ and penicillin $(51 \cdot 3 \%, 268)$. The penicillin-nonsusceptible strains accounted for $67 \cdot 8 \%$ of all strains. However, the isolates were found to exhibit high susceptibility to ceftriaxone $(92 \cdot 9 \%, 485)$, ofloxacin $(87 \cdot 0 \%, 454)$, cefotaxime $(85 \cdot 8 \%, 448)$ and chloramphenicol $(72 \cdot 0 \%$, $376)$. No vancomycin-resistant isolates were detected. Significantly more isolates from children under 14 years showed high resistance to penicillin than isolates from adults above 14 years $(67 \cdot 4$ and $44 \cdot 4 \%$, respectively).

Table 3 summarizes the predominant serotypes of $S$. pneumoniae resistant to penicillin. Among the seven major serotypes, $23 \mathrm{~F}$ showed the highest rate $(96 \cdot 2 \%)$ of penicillin resistance, followed by serotypes $19 \mathrm{~F}(77 \cdot 1 \%), 6 \mathrm{~B}(72 \cdot 1 \%)$, $9 \mathrm{~V}(68 \cdot 4 \%), 14(59 \cdot 4 \%), 4(23 \cdot 1 \%)$ and $3(4 \cdot 2 \%)$. Three of the serotype 3 strains were found to be penicillinresistant.

The antibiotic-resistance patterns of the isolates to penicillin, cefotaxime, chloramphenicol and erythromycin in relation to the vaccine and non-vaccine serotypes are summarized in Table 4 . Of the invasive isolates, $27 \cdot 6 \%$ $(144 / 522)$ were resistant to three or four kinds of antimicrobials and $45.0 \%(235 / 522)$ were dual resistant. The proportion of serotype $23 \mathrm{~F}$ strains exhibiting resistance to three or more antibiotics was significantly high $(62 \cdot 1 \%$ for four antibiotics and $25 \cdot 2 \%$ for three antibiotics, respectively). Serotype $6 \mathrm{~B}$ also demonstrated a high proportion of triple resistance. Among serotype 3 strains, resistance to two or three antibiotics was quite frequent $(68 \cdot 1 \%, 49 / 72)$, but no strain exhibited multiple resistance to all four antibiotics. The 7-, 11- and 23-valent vaccines can cover over $80 \%$ of the multiple-drug-resistant strains.

\section{DISCUSSION}

S. pneumoniae can cause invasive infections with high morbidity and mortality in all ages, including symptoms of pneumonia, bacteraemia and meningitis. Risk groups for serious invasive pneumococcal infections are children ( $<2$ years), the elderly ( $>65$ years) and immunocompromised individuals (Schreiber \& Jacobs, 1995). The emerging resistance to penicillin and other commonly used antibiotics has revived interest in the prevention of pneumococcal disease by vaccination (Bogaert et al., 2004). Continuing surveillance of serotype distribution and antibiotic resistance of clinical isolates is importance for vaccine evaluation.

According to our surveillance data on 28 hospitals, people over 65 years of age and young children under 2 years old were the major high-risk groups for S. pneumoniae infection. This age distribution is similar to that of other countries (Luey \& Kam, 1996).

Twenty-six different invasive S. pneumoniae serotypes were identified in Taiwan in this study. The most frequently 
Table 2. Susceptibility of isolates from patients aged less than or older than 14 years in Taiwan, 2002-2003, to nine antimicrobial agents

\begin{tabular}{|c|c|c|c|c|c|c|c|c|c|}
\hline \multirow[t]{3}{*}{ Antibiotic } & \multicolumn{9}{|c|}{ No. of isolates (\%) } \\
\hline & \multicolumn{3}{|c|}{ All age groups } & \multicolumn{3}{|c|}{ Under 14 years of age } & \multicolumn{3}{|c|}{ Older than 14 years of age } \\
\hline & Sensitive & Intermediate & Resistant & Sensitive & Intermediate & Resistant & Sensitive & Intermediate & Resistant \\
\hline Penicillin & $168(32 \cdot 2)$ & $86(16 \cdot 5)$ & $268(51 \cdot 3)$ & $24(16 \cdot 3)$ & $24(16 \cdot 3)$ & $99(67 \cdot 4)^{*}$ & $140(39 \cdot 3)$ & $58(16 \cdot 3)$ & $158(44 \cdot 4)^{\star}$ \\
\hline Cefotaxime & $448(85 \cdot 8)$ & $67(12 \cdot 9)$ & $7(1 \cdot 3)$ & $114(77 \cdot 6)$ & $30(20 \cdot 4)$ & $3(2 \cdot 0)$ & $319(89 \cdot 6)$ & $33(9 \cdot 3)$ & $4(1 \cdot 1)$ \\
\hline Ceftriaxone & $485(92 \cdot 9)$ & $33(6 \cdot 3)$ & $4(0 \cdot 8)$ & $133(90 \cdot 5)$ & $13(8 \cdot 8)$ & $1(0 \cdot 7)$ & $334(93 \cdot 8)$ & $19(5 \cdot 4)$ & $3(0 \cdot 8)$ \\
\hline Chloramphenicol & $376(72 \cdot 0)$ & 0 & $146(28 \cdot 0)$ & $106(72 \cdot 1)$ & 0 & $41(27 \cdot 9)$ & $257(72 \cdot 2)$ & 0 & $99(27 \cdot 8)$ \\
\hline Erythromycin & $82(15 \cdot 7)$ & $31(5 \cdot 9)$ & $409(78 \cdot 4)$ & $9(6 \cdot 1)$ & $9(6 \cdot 1)$ & $129(87 \cdot 8)^{*}$ & $69(19 \cdot 4)$ & $20(5 \cdot 6)$ & $267(75 \cdot 0)^{\star}$ \\
\hline Ofloxacin & $454(87 \cdot 0)$ & $60(11 \cdot 5)$ & $8(1 \cdot 5)$ & $136(92 \cdot 5)$ & $9(6 \cdot 1)$ & $2(1 \cdot 4)$ & $303(85 \cdot 1)$ & $48(13 \cdot 5)$ & $5(1 \cdot 4)$ \\
\hline Tetracycline & $58(11 \cdot 1)$ & $22(4 \cdot 2)$ & $442(84 \cdot 7)$ & $15(10 \cdot 2)$ & $8(5 \cdot 4)$ & $124(84 \cdot 4)$ & $41(11 \cdot 5)$ & $14(3 \cdot 9)$ & $301(84 \cdot 6)$ \\
\hline TMP/SMX† & $158(30 \cdot 3)$ & $91(17 \cdot 4)$ & $273(52 \cdot 3)$ & $28(19 \cdot 1)$ & $24(16 \cdot 3)$ & $95(64 \cdot 6)^{*}$ & $124(34 \cdot 8)$ & $66(18 \cdot 6)$ & $166(46 \cdot 6)^{\star}$ \\
\hline Vancomycin & $522(100)$ & 0 & 0 & $147(100)$ & 0 & 0 & $356(100)$ & 0 & 0 \\
\hline
\end{tabular}

*Significant differences between two age groups in resistance $(\mathrm{OR}=2 \cdot 51, P=0 \cdot 00001$ for penicillin; $\mathrm{OR}=2 \cdot 42, P=0 \cdot 0017$ for erythromycin; $\mathrm{OR}=2 \cdot 01, P=0 \cdot 0007$ for trimethoprim/sulfamethoxazole).

$\dagger$ TMP/SMX, trimethoprim/sulfamethoxazole.

isolated serotypes of S. pneumoniae were 14, 23F, 3, 19F, 6B and $9 \mathrm{~V}$, in decreasing order of frequency. Serotype distribution in age groups above and below 14 years was similar, with only slight differences in rank order, in particular for type 3, which was more prevalent in adults aged over 14 years. However, the overall frequency of isolation of serotype 3 was higher than in other countries (Denham \& Clarke, 2005; Reinert et al., 2005), except in Japan and Italy (Kasahara et al., 2005; Marchese et al., 2005). More isolates from children younger than 14 years old were resistant to penicillin than from adults over 14 years and this was statistically significant. After further analysis, the

Table 3. Susceptibility to penicillin of different serotypes of invasive $S$. pneumoniae in Taiwan

\begin{tabular}{|lcccc|}
\hline \multirow{2}{*}{ Serotype } & \multirow{2}{*}{$\begin{array}{c}\text { No. of } \\
\text { isolates }\end{array}$} & \multicolumn{3}{c|}{ Penicillin susceptibility $(\%)$} \\
\cline { 3 - 5 } & & Sensitive & Intermediate & Resistant \\
\hline 14 & 96 & $10(10 \cdot 4)$ & $29(30 \cdot 2)$ & $57(59 \cdot 4)$ \\
$23 \mathrm{~F}$ & 79 & $1(1 \cdot 3)$ & $2(2 \cdot 5)$ & $76(96 \cdot 2)^{*}$ \\
3 & 72 & $53(73 \cdot 6)$ & $16(22 \cdot 2)$ & $3(4 \cdot 2)^{*}$ \\
$19 \mathrm{~F}$ & 70 & $6(8 \cdot 6)$ & $10(14 \cdot 3)$ & $54(77 \cdot 1)^{*}$ \\
$6 \mathrm{~B}$ & 43 & $5(11 \cdot 6)$ & $7(16 \cdot 3)$ & $31(72 \cdot 1)^{*}$ \\
$9 \mathrm{~V}$ & 19 & $3(15 \cdot 8)$ & $3(15 \cdot 8)$ & $13(68 \cdot 4)$ \\
4 & 13 & $9(69 \cdot 2)$ & $1(7 \cdot 7)$ & $3(23 \cdot 1)$ \\
11 & 10 & $9(90 \cdot 0)$ & 0 & $1(10 \cdot 0)$ \\
Others & 120 & $72(60 \cdot 0)$ & $18(15 \cdot 0)$ & $30(25 \cdot 0)$ \\
& & & & \\
\hline
\end{tabular}

${ }^{\star}$ Significant differences in penicillin resistance $(\mathrm{OR}=36 \cdot 23, \quad P=$ $0 \cdot 0000001$ for serotype $23 \mathrm{~F}$; $\mathrm{OR}=4 \cdot 13, P=0.0000001$ for serotype 19F; $\mathrm{OR}=2 \cdot 67, \quad P=0.005$ for serotype $6 \mathrm{~B}$; and $\mathrm{OR}=0.03, P=$ $0 \cdot 00000001$ for serotype 3 ). discrepancy of penicillin resistance was found to be due to the different prevalence rates of serotype 3 isolates between these two age groups $(6 \cdot 1 \%$ in the group $\leqslant 14$ years old, $17 \cdot 4 \%$ in the group $>14$ years old), because the penicillin-non-susceptibility rate is low in serotype 3 isolates. Mortality among patients $\geqslant 65$ years old was higher than in the other age groups. The mortality rate of serotype 3 and $6 \mathrm{~B}$ in the elderly ( $>65$ years) was 20 and $50 \%$, respectively. Among the most prevalent serotypes, the crude mortality rates of serotype $6 \mathrm{~B}$ and 3 were $13 \cdot 3$ and $20.0 \%$, respectively; both rates were about twice those of serotypes $14,23 \mathrm{~F}$ and $19 \mathrm{~F}(12 \cdot 0,10 \cdot 7$ and $12 \cdot 0 \%$, respectively). In this study, $93 \cdot 0$ and $2 \cdot 3 \%$ of serotype $6 \mathrm{~B}$ strains were isolated from blood and cerebrospinal fluid, respectively. Higher association with invasive disease has been reported for serotype 6B (Hanage et al., 2005). Whether the higher mortality can be attributed to the higher invasiveness of serotypes 3 and $6 \mathrm{~B}$ or to stronger antibiotic resistance of serotype $6 \mathrm{~B}$ deserves further investigation.

The coverage of the 23-valent polysaccharide vaccine and 7valent conjugate pneumococcal vaccine for all age groups was $87 \cdot 2 \%(455 / 522)$ and $63 \cdot 0 \%(329 / 522)$, respectively. For the elderly aged 65 years and over, the coverage of the 23 -valent polysaccharide vaccine was $88 \cdot 5 \%$ (169/191). In children under 2 years of age, the coverage rate of the 23valent vaccine was as high as $88 \cdot 5 \%(46 / 52)$; however, the protection from the 23 -valent vaccine is not sufficient and the 7-valent conjugate vaccine has to be used instead. For infants under 2 years old, the coverage of the 7- and 11valent conjugate pneumococcal vaccines was $78 \cdot 8 \%(41 / 52)$ and $86.5 \%(45 / 52)$, respectively. The vaccination rate of pneumococcal vaccine in Taiwan has been quite low; whether this can explain the high prevalence of vaccinecovered serotypes deserves further study. 
Between 1999 and 2003, the prevalence rate of penicillin non-susceptibility varied from 38 to $81 \%$ worldwide (Schito \& Felmingham, 2005). In a previous report, the prevalence rate $(60-84 \%)$ of clinical penicillin-non-susceptible isolates was remarkably high in Taiwan between 1981 and 2000 (Hsueh et al., 2002). Although the policy for the restrictive use of antibiotics began in 2001, the penicillin-resistance rate of $S$. pneumoniae was still high in this study. Analysis of the antimicrobial-resistance results showed that there was a high prevalence of $\beta$-lactam- and macrolide-resistant $S$. pneumoniae in Taiwan. Penicillin is still the major antibiotic used to treat infection of invasive S. pneumoniae. Our surveillance study of $S$. pneumoniae in Taiwan revealed that only $32 \cdot 2 \%(168 / 522)$ of the isolates were susceptible to penicillin, results similar to those of a study conducted in 2000-2001 that also surveyed S. pneumoniae resistance in Taiwan (Hsueh et al., 2003). The MIC analysis clearly showed that serotypes $23 \mathrm{~F}, 19 \mathrm{~F}, 6 \mathrm{~B}, 9 \mathrm{~V}$ and 14 generally were not susceptible to penicillin. Thus, antibiotic selection pressures may have resulted in serotypes 23F, 19F, 6B, $9 \mathrm{~V}$ and 14 becoming more prevalent (Doern, 2001). For serotype 3, strains resistant to penicillin have rarely been found (Kasahara et al., 2005). In our study, three penicillinresistant isolates were discovered in serotype 3 pneumococci. All three penicillin-resistant isolates were also resistant to erythromycin and tetracycline. Two of the three penicillinresistant serotype 3 isolates exhibited an identical PFGE pattern, which was similar to that of other penicillinresistant and erythromycin-susceptible serotype 3 isolates and belonged to multilocus sequence type ST180. The PFGE pattern of the other penicillin-resistant type 3 isolate was quite distinct and different from that of all other type 3 isolates (data not shown). Macrolide-resistant ST180 of serotype 3 has been reported to be prevalent in many Asian countries such as Hong Kong and Japan (Bozdogan et al., 2004; Kasahara et al., 2005). It is possible that these penicillin-resistant serotype 3 clones have evolved through recombination with other resistant $S$. pneumoniae clones or were introduced into Taiwan very recently. Of the invasive isolates, $88.7 \%$ were resistant to at least one of the four antibiotics (penicillin, cefotaxime, chloramphenicol and erythromycin). Strains of five of the most prevalent serotypes $(14,23 \mathrm{~F}, 19 \mathrm{~F}, 6 \mathrm{~B}, 3)$ accounted for $74 \cdot 3 \%$ of strains resistant to these four antibiotics. Of the invasive isolates, $72.6 \%$ were resistant to at least two antibiotics. The proportion of serotype $23 \mathrm{~F}$ strains exhibiting multiple drug resistance was extremely high ( $98 \cdot 7 \%)$. Serotypes 14,19 and $6 \mathrm{~B}$ also demonstrated a high proportion of multiple drug resistance. Among serotype 3 strains, resistance to more than two antibiotics other than penicillin was quite high. The 7-, 11- and 23 -valent vaccines can cover over $70 \%$ of the multiple-drug-resistant strains.

In conclusion, the current vaccines can cover approximately $80 \%$ of the invasive strains in the target age groups and the multiple-drug-resistant strains. The occurrence or possible introduction of new clones such as the multiple-resistant type 3 strains into Taiwan and the impact of this should be 
monitored. Continuous surveillance is important for elaboration of the control and immunization policy of Taiwan.

\section{ACKNOWLEDGEMENTS}

This work was in part supported by grants DOH91-DC-2023 and DOH92-DC-2014 from the Center for Disease Control, Department of Health, Taiwan. We would like to express our sincere appreciation to all of the participants in the hospital microbiology laboratories for providing the strains and information related to these strains. They are the Tri Service General Hospital, Buddhist Tzu Chi General Hospital at Da-Lin, St Martin De Porres Hospital, Taichung Veterans General Hospital, Taipei Municipal Jen-Ai Hospital, Taipei Municipal Hoping Hospital, Taiwan Adventist Hospital, Kuang Tien General Hospital at Ta-Chia, Kuang Tien General Hospital at Sha-Lu, Mennonite Christian Hospital, Kaohsiung Chang Gung Memorial Hospital, Keelung Chang Gung Memorial Hospital, Jen-Ai Hospital Tali Branch, St Joseph's Hospital, Mackay Memorial Hospital main branch, Kaohsiung Veterans General Hospital, Zouying Armed Forces Hospital, KangNing General Hospital, Mackay Memorial Hospital Tamshui branch, Hsinchu Mackay Memorial Hospital, Hualien Hospital Department of Health, Hsinchu General Hospital Department of Health, Chia-Yi Christian Hospital, Changhua Christian Hospital and Lo-Tung Poh Ai Hospital.

\section{REFERENCES}

Baltimore, R. S. (1992). New challenges in the development of a conjugate pneumococcal vaccine. JAMA (J Am Med Assoc) 268, 3366-3367.

Bogaert, D., Hermans, P. W. M., Adrian, P. V., Rümke, H. C. \& de Groot, R. (2004). Pneumococcal vaccines: an update on current strategies. Vaccine 22, 2209-2220.

Bozdogan, B., Bogdanovich, T., Kosowska, K., Jacobs, M. R. \& Appelbaum, P. C. (2004). Macrolide resistance in Streptococcus pneumoniae: clonality and mechanisms of resistance in 24 countries. Curr Drug Targets Infect Disord 4, 169-176.

Chen, J.-Y., Fung, C. P., Wang, C. C., Chu, M. L. \& Siu, L. K. (2003). In vitro susceptibility of six fluoroquinolones against invasive Streptococcus pneumoniae isolated from 1996 to 2001 in Taiwan. Microb Drug Resist 9, 211-217.

Chiou, C.-C. C., Liu, Y.-C., Huang, T.-S., Hwang, W.-K., Wang, J.-H., Lin, H.-H., Yen, M.-Y. \& Hsieh, K.-S. (1998). Extremely high prevalence of nasopharyngeal carriage of penicillin-resistant Streptococcus pneumoniae among children in Kaohsiung, Taiwan. J Clin Microbiol 36, 1933-1937.

Denham, B. C. \& Clarke, S. C. (2005). Serotype incidence and antibiotic susceptibility of Streptococcus pneumoniae causing invasive disease in Scotland, 1999-2002. J Med Microbiol 54, 327-331.

Doern, G. V. (2001). Antimicrobial use and the emergence of antimicrobial resistance with Streptococcus pneumoniae in the United States. Clin Infect Dis 33, S187-S192.

Hanage, W. P., Kaijalainen, T. H., Syrjänen, R. K., Auranen, K., Leinonen, M., Mäkelä, P. H. \& Spratt, B. G. (2005). Invasiveness of serotypes and clones of Streptococcus pneumoniae among children in Finland. Infect Immun 73, 431-435.

Hausdorff, W. P., Feikin, D. R. \& Klugman, K. P. (2005). Epidemiological differences among pneumococcal serotypes. Lancet Infect Dis 5, 83-93.
Hsueh, P.-R., Wu, J.-J. \& Hsiue, T.-R. (1996). Invasive Streptococcus pneumoniae infection associated with rapidly fatal outcome in Taiwan. J Formos Med Assoc 95, 364-371.

Hsueh, P.-R., Liu, C.-Y. \& Luh, K.-T. (2002). Current status of antimicrobial resistance in Taiwan. Emerg Infect Dis 8, 132-137.

Hsueh, P.-R., Teng, L.-J., Wu, T.-L. \& 17 other authors (2003). Telithromycin- and fluoroquinolone-resistant Streptococcus pneumoniae in Taiwan with high prevalence of resistance to macrolides and $\beta$-lactams: SMART program 2001 data. Antimicrob Agents Chemother 47, 2145-2151.

Kasahara, K., Maeda, K., Mikasa, K. \& 7 other authors (2005). Clonal dissemination of macrolide-resistant and penicillinsusceptible serotype 3 and penicillin-resistant Taiwan 19F-14 and 23F-15 Streptococcus pneumoniae isolates in Japan: a pilot surveillance study. J Clin Microbiol 43, 1640-1645.

Lo, W.-T., Wang, C.-C., Yu, C.-M. \& Chu, M.-L. (2003). Rate of nasopharyngeal carriage, antimicrobial resistance and serotype of Streptococcus pneumoniae among children in northern Taiwan. J Microbiol Immunol Infect 36, 175-181.

Luey, K. Y. \& Kam, K. M. (1996). Vaccine coverage of Streptococcus pneumoniae in Hong Kong with attention to the multiple-antibioticresistant strains. Vaccine 14, 1573-1580.

Marchese, A., Gualco, L., Cochetti, I., Montanari, M. P., Speciale, A. M., Musumeci, S. R., Varaldo, P. E., Nicoletti, G. \& Schito, G. C. (2005). Antibiotic susceptibility and serotype distribution in Streptococcus pneumoniae circulating in Italy: results of the SEMPRE surveillance study (2000-2002). Int J Antimicrob Agents 26, 138-145.

Reinert, R. R., Reinert, S., van der Linden, M., Cil, M. Y., AlLahham, A. \& Appelbaum, P. (2005). Antimicrobial susceptibility of Streptococcus pneumoniae in eight European countries from 2001 to 2003. Antimicrob Agents Chemother 49, 2903-2913.

Robbins, J. B. \& Schneerson, R. (1990). Polysaccharide-protein conjugates: a new generation of vaccines. J Infect Dis 161, 821-832.

Schito, G. C. \& Felmingham, D. (2005). Susceptibility of Streptococcus pneumoniae to penicillin, azithromycin and telithromycin (PROTEKT 1999-2003). Int J Antimicrob Agents 26, 479-485.

Schreiber, J. R. \& Jacobs, M. R. (1995). Antibiotic-resistant pneumococci. Pediatr Clin North Am 42, 519-537.

Shi, Z.-Y., Enright, M. C., Wilkinson, P., Griffiths, D. \& Spratt, B. G. (1998). Identification of three major clones of multiply antibioticresistant Streptococcus pneumoniae in Taiwanese hospitals by multilocus sequence typing. J Clin Microbiol 36, 3514-3519.

Siu, L. K., Chu, M.-L., Ho, M., Lee, Y.-S. \& Wang, C.-C. (2002). Epidemiology of invasive pneumococcal infection in Taiwan: antibiotic resistance, serogroup distribution, and ribotypes analyses. Microb Drug Resist 8, 201-208.

Song, J.-H., Jung, S.-I., Ko, K.-S. \& 24 other authors (2004). High prevalence of antimicrobial resistance among clinical Streptococcus pneumoniae isolates in Asia (an ANSORP study). Antimicrob Agents Chemother 48, 2101-2107.

Whitney, C. G., Farley, M. M., Hadler, J. \& 9 other authors (2000). Increasing prevalence of multidrug-resistant Streptococcus pneumoniae in the United States. N Engl J Med 343, 1917-1924.

Whitney, C. G., Farley, M. M., Hadler, J. \& 10 other authors (2003). Decline in invasive pneumococcal disease after the introduction of protein-polysaccharide conjugate vaccine. $N$ Engl J Med 348, $1737-1746$. 